\title{
ON THE ORDER OF ACCURACY OF FINITE DIFFERENCE OPERATORS ON DIAGONAL NORM BASED SUMMATION-BY-PARTS FORM*
}

\author{
VIKTOR LINDERS ${ }^{\dagger}$, TOMAS LUNDQUIST ${ }^{\dagger}$, AND JAN NORDSTRÖM $^{\dagger}$
}

\begin{abstract}
In this paper we generalize results regarding the order of accuracy of finite difference operators on summation-by-parts (SBP) form, previously known to hold on uniform grids, to grids with arbitrary point distributions near domain boundaries. We give a definite proof that the order of accuracy in the interior of a diagonal norm based SBP operator must be at least twice that of the boundary stencil, irrespective of the grid point distribution near the boundary. Additionally, we prove that if the order of accuracy in the interior is precisely twice that of the boundary, then the diagonal norm defines a quadrature rule of the same order as the interior stencil. Again, this result is independent of the grid point distribution near the domain boundaries.
\end{abstract}

Key words. finite difference schemes, summation-by-parts operators, numerical differentiation, quadrature rules, order of accuracy

AMS subject classifications. $65 \mathrm{M} 06,65 \mathrm{~N} 06$

DOI. $10.1137 / 17 \mathrm{M} 1139333$

1. Introduction. Summation-by-parts (SBP) operators, applied in the discretization of systems of partial differential equations, have received considerable attention since they lead to provable energy stability [27] and, recently, entropy stability [6] for well-posed problems. Finite difference stencils on SBP form were first introduced in $[14,15]$ based on central difference schemes of orders 2 and 4 . Later, operators with minimal bandwidth using stencils of orders 6 and 8 were developed in [24]. In [3, 21] SBP operators of orders up to 8 for both first and second derivatives were presented.

The SBP concept has been extended to methods outside the finite difference community. These include spectral collocation and spectral element methods $[2,8,9]$ as well as correction procedures via reconstruction [23]. Further, the finite difference class of SBP operators has been enlarged to multidimensional operators similar to Galerkin methods [12] as well as to grid dependent stencils akin to element based methods [5]. In this paper we restrict our attention to fixed stencil finite difference schemes and do not consider these extended approaches further.

Implicit to the definition of an SBP operator is the notion of a discrete norm. If this norm is represented by a diagonal matrix, the associated operator is referred to as a diagonal norm based SBP operator. To avoid stability issues on curvilinear grids [25], and in general for problems with variable coefficients [22], finite difference operators on SBP form are in practice usually based on a diagonal norm.

The focus in this paper is on SBP operators consisting of a repeated central difference stencil in the interior and one-sided stencils near boundaries and interfaces. We introduce the notation $\operatorname{SBP}(\tau, 2 s)$ to refer to such an operator that is of order $\tau$ near the boundary and of order $2 s$ in the interior. For completely uniform grid distributions, the accuracy of $\operatorname{SBP}(\tau, 2 s)$ is known to be dictated by two main constraints; we will formalize these as Theorems 4 and 5 in the next section. The first one states

${ }^{*}$ Received by the editors July 18, 2017; accepted for publication (in revised form) March 15, 2018; published electronically April 17, 2018.

http://www.siam.org/journals/sinum/56-2/M113933.html

$\dagger^{\dagger}$ Department of Mathematics, Computational Mathematics, Linköping University, SE-581 83 Linköping, Sweden (viktor.linders@liu.se, tomas.lundquist@liu.se, jan.nordstrom@liu.se).

1048 
that $s \geq \tau$, i.e., the order of accuracy of the interior stencil is at least twice that of the boundary [14]. This implies a global convergence rate of order $\tau+1$ for first order hyperbolic problems, and $\tau+2$ for parabolic and second order hyperbolic problems, if the approximation is pointwise bounded [10,11, 26, 28]. Naturally, this reduction from the design order $2 s$ is a significant drawback of the diagonal norm based high order SBP approach.

The second theorem states that if $s=\tau$, the diagonal norm defines a quadrature rule of order $2 \tau$ [13]. This result was shown in [18] to have a profound impact on the accuracy of SBP preserving interpolation operators [20], used for multiblock couplings with noncollocated interfaces, leading to truncation errors of order $\tau-1$ for hyperbolic problems.

Suggestions for overcoming the order restrictions that follow from Theorems 4 and 5 have recently been presented in [5, 7]. These approaches are able to preserve the design order of accuracy for SBP operators near boundaries and in the presence of nonconforming grid interpolation. A drawback of these methods is that the coefficients of the resulting operators are explicitly grid size dependent, and hence a new operator must be constructed for each grid. As such, these operators are akin to spectral element methods rather than finite differences, and we shall not consider them further in this paper.

Theorems 4 and 5 both assume that a uniform grid that conforms with the physical domain boundaries is used. However, in recent years, finite difference operators on SBP form defined on grids that do not match with the physical domain boundaries have been introduced [4]. Further, operators defined on grids with nonuniform point distributions near boundaries and interfaces have emerged [19]. Significant error reductions have been observed for several model problems using such operators.

In [19], SBP operators with up to eight boundary stencils and three nonuniform grid points were constructed without an increase in the formal order of accuracy near the boundary. This gives an indication that Theorem 4 may hold also in the nonuniform setting, though due to the limited parameter space, it does not constitute a proof. The purpose of this paper is to provide formal proofs of the generalizations of Theorems 4 and 5 to grids with arbitrary nonuniform point distributions near boundaries and interfaces, where in addition we do not require the grid points to match with physical domain boundaries. The results hold irrespective of the number of nonuniform grid points, the dimension of the boundary stencils, and the width of the interior stencils. The implication of these results is clear: approaches such as the one in $[19]$ or $[17,16]$, where wider interior and boundary stencils are used to minimize numerical errors, cannot increase the formal order of accuracy of diagonal norm based SBP operators.

The remainder of the paper is structured as follows. In section 2 we formalize the definition of finite difference stencils on SBP form as well as the classical accuracy results for uniform grids. We devote section 3 to studying the properties of the diagonal norm implicit to the definition of the SBP operators. We utilize a dual interpretation of the norm, on one hand as an integral part of an SBP operator and on the other as a freestanding high order quadrature rule. In this way we obtain two separate sets of conditions that must be simultaneously satisfied by the matrix coefficients. In section 4 we use these conditions to prove a generalized version of Theorem 4. In section 5 we show that a generalization of Theorem 5 follows in a straightforward fashion. Finally, conclusions are drawn in section 6 . 
2. Preliminaries. Before we proceed we will specify what we mean when we refer to a finite difference operator on SBP form with a prescribed order of accuracy. With such specifications in place we may concisely state the classical theorems that will be the subjects of generalization later in this paper.

2.1. Definitions and notation. We will henceforth consider the real, finite interval $[a, b]$. Upon discretizing the interval by projecting it onto a discrete grid vector $\mathbf{x}$, we do not assume that the grid points match with the domain boundaries $x=a$ and $x=b$. The grids we will consider are of the form

$$
\mathbf{x}=\left(x_{0}, x_{1}, \ldots, x_{r}, x_{r}+\Delta x, x_{r}+2 \Delta x, \ldots\right)^{T},
$$

where $x_{0}<x_{1}<\cdots<x_{r}$ and $x_{r}$ is the final point in the region of $\mathbf{x}$ with arbitrary grid spacings. Beyond this point, the grid is necessarily uniform with grid spacing $\Delta x$. An example where $r=5$ is shown in Figure 1. Here $a<x_{0}$; however, this is not a requirement.

Definition 1. A matrix $D=P^{-1} Q$ defined on the grid $\mathbf{x}$ is said to have the SBP property if

1. $P=P^{T}>0$,

2. $\left(Q+Q^{T}\right)=e_{b}^{T} e_{b}-e_{a}^{T} e_{a}$,

where $P$ and $Q$ are square $(N+1) \times(N+1)$ matrices and $e_{a, b}$ are boundary interpolation operators defined on $\mathbf{x}$, i.e., row vectors of dimension $1 \times(N+1)$.

Definition 2. An SBP operator $D$ defined on the grid $\mathbf{x}$ is said to be accurate to order $\tau \geq 1$ if it satisfies

1. $D \mathbf{x}^{j}=j \mathbf{x}^{j-1}, j=0, \ldots, \tau$,

2. $e_{a} \mathbf{x}^{j}=a^{j}, e_{b} \mathbf{x}^{j}=b^{j} \quad j=0, \ldots, \tau$.

Remark 1. In [4] the accuracy of the boundary interpolation operators is taken to be greater than or equal to $\tau$. Such interpolation operators satisfy the requirements of Definition 2 and may therefore be considered special cases of the setting considered here. Increasing the accuracy of the interpolation operators does not affect the validity of the results from the forthcoming analysis.

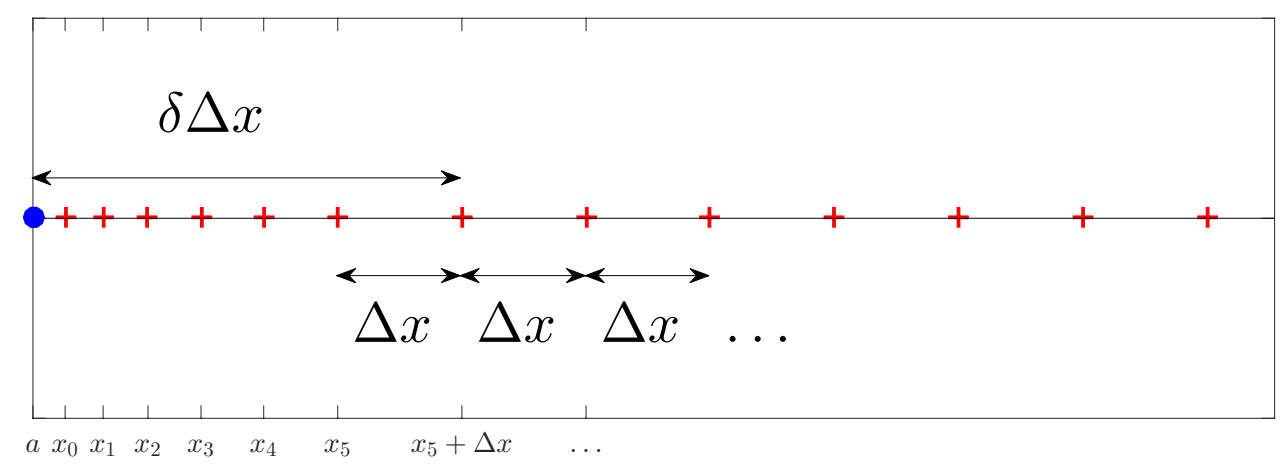

FIG. 1. Example of a nonuniform grid with $r=5$ arbitrary grid points near the left boundary. Here, $\delta \Delta x$ denotes the distance between the physical boundary and the point at which the stencil becomes uniform.

Copyright $@$ by SIAM. Unauthorized reproduction of this article is prohibited. 
By Taylor's formula, Definition 2 implies that the truncation error $\left\|D \mathbf{f}-\mathbf{f}^{\prime}\right\|_{\infty}$ vanishes at a rate $\mathcal{O}\left(\Delta x^{\tau}\right)$ as $\Delta x \rightarrow 0$. Here, $\mathbf{f}$ and $\mathbf{f}^{\prime}$ are projections of the smooth function $f(x)$ and its derivative $f^{\prime}(x)$ onto the computational grid.

Remark 2. Here and henceforth, for any $j \geq 0, \mathbf{x}^{j}$ is to be understood as the elementwise exponentiation of the vector $\mathbf{x}$. We use the interpretation $\mathbf{x}^{-1}=\mathbf{0}$ and $0^{0}=1$ where necessary.

Remark 3. Typically, if matching grid points are used at the domain boundaries, i.e., $x_{0}=a$ and $x_{N}=b$, the interpolation operators take the specific form $e_{a}=$ $(1,0, \ldots, 0)$ and $e_{b}=(0, \ldots, 0,1)$. It follows that $Q+Q^{T}=\operatorname{diag}(-1,0, \ldots, 0,1)$.

In Definitions 1 and 2, $\tau$ refers to the accuracy at the boundary of the operator. In the interior, a finite difference SBP operator may be, and generally is, more accurate. What we mean by the boundary and the interior will be made precise in due course.

With a slight abuse of notation, we refer to the matrix $P$ in Definition 1 as the norm since it defines a discrete approximation of the $L^{2}$-norm on $[a, b]$ :

$$
\|\mathbf{u}\|_{P}=\left(\mathbf{u}^{T} P \mathbf{u}\right)^{1 / 2} \approx \sqrt{\int_{a}^{b} u^{2}(x) \mathrm{d} x} .
$$

Throughout this paper, $P$ will be a diagonal matrix. Hence we will sometimes refer to $P$ as the diagonal norm. As we will see, the norm $P$ defines a high order quadrature rule. An indication that this is the case is given by the so-called compatibility conditions $[14,4,18,12]$ : We multiply the first accuracy condition in Definition 2 from the left by $\left(\mathbf{x}^{i}\right)^{T} P$ to obtain

$$
\left(\mathbf{x}^{i}\right)^{T} Q \mathbf{x}^{j}=j\left(\mathbf{x}^{i}\right)^{T} P \mathbf{x}^{j-1}=j \mathbf{1}_{N}^{T} P \mathbf{x}^{i+j-1}, \quad i, j=0, \ldots, \tau,
$$

where in the last equality we have used the facts that $P$ is diagonal and that $\mathbf{x}^{0}=\mathbf{1}_{N}$, i.e., the $N+1$ point grid vector of all ones. Now swap the indices $i$ and $j$ and add the result to obtain the simplest form of the compatibility conditions;

$$
\left(\mathbf{x}^{i}\right)^{T}\left(Q+Q^{T}\right) \mathbf{x}^{j}=(i+j) \mathbf{1}_{N}^{T} P \mathbf{x}^{i+j-1} .
$$

From Definition 1 and the second accuracy condition in Definition 2 it follows that

$$
(i+j) \mathbf{1}_{N}^{T} P \mathbf{x}^{i+j-1}=b^{i+j}-a^{i+j}, \quad i, j=0, \ldots, \tau .
$$

In other words, $P$ integrates polynomials up to order $2 \tau-1$ exactly. In section 3 we will show that this is equivalent to $P$ defining a quadrature rule of order $2 \tau$. We will therefore sometimes refer to $P$ as the quadrature. In section 5 we show that this is in fact a strict upper bound, i.e., $P$ is not of higher order than $2 \tau$.

2.2. The structure of $\boldsymbol{P}$ and $\boldsymbol{Q}$. As mentioned above, we will restrict our attention to SBP operators based on finite difference stencils. By this we mean that $Q$ is a banded matrix which, away from boundaries, assumes the form of a repeated interior stencil consisting of central finite differences. In order to close the operator near boundaries the stencils are modified and become progressively more one-sided. The most general form of $Q$ near the left boundary is 
(3)

$$
Q=\left(\begin{array}{cccccccccc}
q_{0,0} & \ldots & q_{0, r} & \ldots & q_{0, r+n-1} & & & & \\
\vdots & \ddots & \vdots & & \vdots & & & & \\
q_{r, 0} & \ldots & q_{r, r} & \ldots & q_{r, r+n-1} & a_{n} & & & \\
\vdots & & \vdots & \ddots & \vdots & \vdots & \ddots & & & \\
q_{r+n-1,0} & \ldots & q_{r+n-1, r} & \ldots & q_{r+n-1, r+n-1} & a_{1} & \ldots & a_{n} & & \\
& & -a_{n} & \ldots & -a_{1} & 0 & a_{1} & \ldots & a_{n} & \\
& & & \ddots & & \ddots & \ddots & \ddots & & \ddots
\end{array}\right) .
$$

Here, $q_{i j}$ are the stencil coefficients of the boundary blocks. The parameters $a_{1}, \ldots, a_{n}$ are the coefficients of the repeated central difference stencil, defined to be of order $2 s$ through the conditions

$$
\frac{\partial u}{\partial x}=\frac{1}{\Delta x} \sum_{k=1}^{n} a_{k}(u(x+k \Delta x, t)-u(x-k \Delta x, t))+\mathcal{O}\left(\Delta x^{2 s}\right) .
$$

Here, $\Delta x$ is the spatial increment of a uniform grid, and $s \geq 1$. A Taylor expansion of the right-hand side of (4) reveals that this is equivalent to the following accuracy conditions:

$$
\sum_{k=1}^{n} a_{k} k^{\mu}= \begin{cases}\frac{1}{2} & \text { if } \mu=1 \\ 0 & \text { if } \mu=3,5, \ldots, 2 s-1\end{cases}
$$

The corresponding conditions with even $\mu$ are automatically satisfied due to the symmetry of the stencil. Note that the interior stencil by definition operates on a uniform grid. The form of $Q$ in (3) is consistent with an arbitrary placing of the first $r$ grid points in $\mathbf{x}$ since none of these points are utilized by the interior stencil. However, since the first $r$ grid spacings are completely arbitrary, this formulation also includes the fully uniform grid as a special case.

Analogous to (3), the norm $P$ takes the form of a diagonal matrix

$$
P=\Delta x \operatorname{diag}\left(p_{0}, \ldots, p_{r+n-1}, 1,1, \ldots\right) .
$$

From Definition 1 it is clear that $p_{j}>0, j=0, \ldots, r+n-1$, in order to ensure positive definiteness. Note in (6) that the diagonal elements of $P$ are equal to $\Delta x$ everywhere except in the $r+n$ rows corresponding to the boundary block of $Q$.

The distinction in structure and accuracy between the boundary and interior of a finite difference SBP operator motivates the following nomenclature.

DeFINITION 3. The finite difference operator $D$ is denoted $S B P(\tau, 2 s)$ if it has the $S B P$ property in Definition 1, satisfies the accuracy conditions in Definition 2, and has a distinct boundary and interior structure given by (3) and (6) with the interior stencil satisfying (5) for $\mu$ up to $2 s-1$ but not for $\mu=2 s+1$.

Throughout this paper we let the right boundary be treated in the same way as the left one. This simplifies the presentations and proofs of Lemmas 6 and 7 in section 3 without impacting the validity of the results. In practice, the assumption means that $p_{N-j}=p_{j}$ and $q_{N-i, N-j}=-q_{i, j}$. Hence, the grid points are placed symmetrically with respect to the physical domain $[a, b]$, i.e., the distance from $a$ to $x_{j}$ is the same as the distance from $x_{N-j}$ to $b$. With this assumption we may henceforth consider only one boundary in our analysis. 
2.3. Classical results on the order of accuracy of SBP operators. As stated in the introduction, there are two major constraints on the order of accuracy of $\operatorname{SBP}(\tau, 2 s)$. The first result was originally shown in [14] and establishes a relation between the orders of accuracy of the interior stencil and the boundary block of the SBP operator.

TheOREm 4. Let $S B P(\tau, 2 s)$ be defined on a uniform grid. Then $s \geq \tau$.

The implication of Theorem 4 is clear; it is not possible to retain the order of the interior stencil in the entire domain when boundaries (or interfaces) are present. In fact, it is well known that if the approximation is pointwise bounded, the convergence rate is generally $\tau+1$ for first order hyperbolic problems and $\tau+2$ for parabolic and second order hyperbolic problems [26]. For higher order methods, this is a significant reduction from the interior order $2 s$.

The second result relates to the matrix $P$ when viewed as a quadrature rule. It was first shown in [13] and applies to the special case $s=\tau$.

Theorem 5. Let $\operatorname{SBP}(\tau, 2 \tau)$ be based on the diagonal norm $P$ and defined on a uniform grid. Then $P$ defines a quadrature rule of order $2 \tau$.

Here, the statement that $P$ is accurate to order $2 \tau$ means that the error

$$
\mathbf{1}_{N}^{T} P \mathbf{f}-\int_{a}^{b} f(x) \mathrm{d} x
$$

is proportional to $\Delta x^{2 \tau}$ or less, where $\mathbf{f}$ is the projection of a smooth function $f(x)$ onto the grid (1).

In the literature, operators are almost exclusively used where $s=\tau$ since this gives the highest convergence rate possible for a given stencil bandwidth. Thus, in practice, Theorem 5 is almost always satisfied strictly. This observation is important for settings where SBP-preserving interpolation operators (see [20]) are used to couple discrete domains with nonconforming interfaces. In [18] it was shown that Theorem 5 implies that schemes using such operators result in truncation errors of order $\tau-1$ when applied to a first order hyperbolic problem.

Theorems 4 and 5 are the main results that determine the order of accuracy of diagonal norm based SBP operators. However, both results assume the use of uniform grids. With the emergence of diagonal norm based SBP operators defined in nonuniform settings [19,4], generalizations of Theorems 4 and 5 are needed. This is the goal of the next three sections.

3. Conditions on $\boldsymbol{P}$. Hereinafter, we let the domain $[a, b]=[0,1]$. There is no loss of generality in this choice since any other domain is related by an affine transformation. However, this particular choice simplifies the remaining analysis.

As our starting point we return to the previously mentioned dual interpretation of $P$, namely, that we may view $P$ either as a quadrature rule of order at least $2 \tau$ or as a weight matrix in the definition of an SBP operator of order $\tau$. Given the structure of $P$ in (6), it is natural to ask what conditions must be satisfied by the coefficients $p_{0}, \ldots, p_{r+n-1}$ in order to fulfill both these roles. To this end we present two lemmas, one for each interpretation of $P$. For brevity of notation, we introduce the normalized grid points $\hat{x}_{j}=\Delta x^{-1} x_{j}$ and the matrix $\hat{P}=\operatorname{diag}\left(p_{0}, \ldots, p_{r+n-1}\right)$ containing the boundary coefficients of $P$, normalized by $\Delta x$.

Starting with the quadrature interpretation of $P$, the following lemma holds. 
Lemma 6. For any quadrature rule $P$ of the form (6) defined on the grid (1), the compatibility conditions (2) are equivalent to the following conditions on the normalized boundary coefficients:

$$
m \mathbf{1}^{T} \hat{P} \hat{\mathbf{x}}_{1}^{m-1}=B_{m}\left(\hat{x}_{r+n}\right), \quad m=1, \ldots, 2 \tau-1 .
$$

Here, 1 is the $r+n$ point grid vector of all ones, $\hat{\mathbf{x}}_{1}=\left(\hat{x}_{0}, \ldots, \hat{x}_{r+n-1}\right)^{T}, \hat{x}_{r+n}=$ $\hat{x}_{r+n-1}+1$ is the point at which the interior stencil is first applied, and $B_{m}(x)$ denotes the mth Bernoulli polynomial. Further, $P$ is accurate at least to order $2 \tau$, i.e., the error $e_{P}$ is given by

$$
e_{P}:=\mathbf{1}_{N}^{T} P \mathbf{f}-\int_{0}^{1} f(x) d x=\mathcal{O}\left(\Delta x^{2 \tau}\right),
$$

where $\mathbf{f}$ is the projection of a smooth function $f(x)$ onto the grid (1).

Proof. It was shown in [1] that a quadrature rule $P$ of the form (6) and defined on $\mathbf{x}$ satisfies the error equation

$$
e_{P}=\sum_{k=1}^{\infty} \Delta x^{k} \frac{f^{(k-1)}(0)+(-1)^{k-1} f^{(k-1)}(1)}{(k-1) !}\left\{\mathbf{1}^{T} \hat{P} \hat{\mathbf{x}}_{1}^{k-1}-\frac{B_{k}\left(\hat{x}_{r+n}\right)}{k}\right\},
$$

where the function $f(x)$ is infinitely differentiable and $\mathbf{f}$ is the projection of $f$ onto the grid $\mathbf{x}$. From (2) we know that $P$ integrates monomials up to order $2 \tau-1$ exactly. Hence, $e_{P}$ vanishes for $f(x)=x^{m-1}, m=1, \ldots, 2 \tau$. By (8), this is equivalent to

$$
\mathbf{1}^{T} \hat{P} \hat{\mathbf{x}}_{1}^{m-1}=\frac{B_{m}\left(\hat{x}_{r+n}\right)}{m}, \quad m=1, \ldots, 2 \tau-1,
$$

where we have noted that when $m=2 \tau$, the term in (8) proportional to $\Delta x^{2 \tau}$ vanishes due to

$$
\frac{f^{(2 \tau-1)}(0)+(-1)^{2 \tau-1} f^{(2 \tau-1)}(1)}{(2 \tau-1) !}=1-1=0
$$

such that (9) need not be satisfied with $m=2 \tau$. The lemma follows.

Details about the Bernoulli polynomials are presented in Appendix A. Next, we consider the SBP interpretation of $P$ and obtain the following lemma.

Lemma 7. For any diagonal norm $P$ of the form (6) defined on the grid (1) and associated with the operator $\operatorname{SBP}(\tau, 2 s)$, the normalized boundary coefficients satisfy the conditions

$$
m \mathbf{1}^{T} \hat{P} \hat{\mathbf{x}}_{1}^{m-1}=\sum_{k=1}^{n} a_{k} \mathcal{P}_{k, m}
$$

for all $m=1, \ldots, 2 \tau$. For each integer $i$ satisfying $\max \{0, m-\tau\} \leq i \leq \min \{m, \tau\}$, the function

$$
\mathcal{P}_{k, m} \equiv \mathcal{P}_{k, m}^{(i)}=\sum_{\nu=1}^{m+1} c_{i, \nu}^{(m)} k^{\nu}
$$

is a polynomial in the summation index $k$ of degree $m+1$. The polynomial coefficients are given by

$$
c_{i, \nu}^{(m)}=\left[1-(-1)^{\nu}\right] B_{m-\nu+1}\left(\hat{x}_{r+n}\right) \sum_{h=0}^{\nu-1}\left(\begin{array}{l}
i \\
h
\end{array}\right) \frac{(-1)^{h}}{m-h+1}\left(\begin{array}{l}
m-h+1 \\
m-\nu+1
\end{array}\right)
$$

and satisfy the following properties: 
(i) Whenever $\nu$ is even, $c_{i, \nu}^{(m)}=0$.

(ii) The first coefficient is given by $c_{i, 1}^{(m)}=2 B_{m}\left(\hat{x}_{r+n}\right)$.

(iii) Whenever $m$ is even, $c_{i, m+1}^{(m)} \neq 0$.

Remark 4. The polynomial $\mathcal{P}_{k, m}^{(i)}$ depends explicitly on the integer $i$; however, the conditions (10) do not. We therefore adopt the simpler notation $\mathcal{P}_{k, m}$ in the remainder while reminding the reader of the dependency on $i$ through the coefficients $c_{i, \nu}^{(m)}$.

The proof of Lemma 7 is extensive and is therefore deferred to Appendix B. There are two main points to note about Lemmas 6 and 7 . The first one is that the left-hand sides of $(7)$ and (10) are equal when $m=1, \ldots, 2 \tau-1$. Of course this implies that the right-hand sides are equal as well. This is the observation from which we will generalize Theorem 4 to the grid (1) in the next section.

The second point to note is that there is one more condition in Lemma 7 than in Lemma 6 , namely, when $m=2 \tau$. As we will see, this extra condition leads directly to a generalization of Theorem 5 .

4. Generalization of Theorem 4. In this section we prove a generalization of Theorem 4 to the nonuniform grid (1). In Lemmas 6 and 7 we have established conditions (7) and (10) on the boundary coefficients of $P$ and naturally these have to be simultaneously satisfied for $m=1, \ldots, 2 \tau-1$. Thus, equating the right-hand sides of (7) and (10), we get

$$
\sum_{k=1}^{n} a_{k} \mathcal{P}_{k, m}=B_{m}\left(\hat{x}_{r+n}\right), \quad m=1, \ldots, 2 \tau-1 .
$$

Note that all quantities involved in (13) are determined by the single grid point $\hat{x}_{r+n}$ and the interior stencil coefficients $a_{k}$. We may thus interpret (13) as a set of conditions on the interior stencil of the SBP operator.

Recall that our goal is to show that the interior stencil (4) is accurate at least to order $2 \tau$. From (5), this means that if we can show that (13) implies

$$
\sum_{k=1}^{n} a_{k} k^{\mu}= \begin{cases}\frac{1}{2} & \text { if } \mu=1, \\ 0 & \text { if } \mu=3,5, \ldots, 2 \tau-1,\end{cases}
$$

then we obtain the generalization of Theorem 4 that we want. In fact, we may prove the following stronger result.

LEMMA 8. The conditions (13) and (14) on the interior stencil are equivalent.

Proof. We begin by showing that (14) implies (13).

Assume that (14) holds. Then, using the definition (11) of $\mathcal{P}_{k, m}$ together with properties (i) and (ii) of the polynomial coefficients, we obtain for $m=1, \ldots, 2 \tau-1$

$$
\sum_{k=1}^{n} a_{k} \mathcal{P}_{k, m}=\underbrace{c_{i, 1}^{(m)} \sum_{k=1}^{n} a_{k} k}_{\begin{array}{c}
=B_{m}\left(\hat{x}_{r+n}\right) \\
\text { by (14) and (ii) }
\end{array}}+\underbrace{c_{i, 3}^{(m)} \sum_{k=1}^{n} a_{k} k^{3}+\cdots+c_{i, m+1}^{(m)} \sum_{k=1}^{n} a_{k} k^{m+1}}_{=0 \text { by (14) and (i) }}=B_{m}\left(\hat{x}_{r+n}\right) .
$$

Thus, (13) is satisfied. Next, we show that (13) implies (14). We do this using induction. Thus, assume that (13) holds for all $\tau$. For $\tau=1$, the left-hand side of (13) with $m=1$ becomes 


$$
\sum_{k=1}^{n} a_{k} \mathcal{P}_{k, 1}=c_{i, 1}^{(1)} \sum_{k=1}^{n} a_{k} k=2 B_{1}\left(\hat{x}_{r+n}\right) \sum_{k=1}^{n} a_{k} k .
$$

The right-hand side of (13) is just $B_{1}\left(\hat{x}_{r+n}\right)$. Thus, it is clear that $\sum a_{k} k=1 / 2$, i.e., (14) holds, whenever $B_{1}\left(\hat{x}_{r+n}\right) \neq 0$. Note that $B_{1}\left(\hat{x}_{r+n}\right)=0$ only in the special case when $\hat{x}_{r+n}=1 / 2$. This corresponds to the situation when the interior stencil is applied only in the middle point of the domain. Such an operator cannot be generalized to an arbitrary number of interior grid points and is therefore not of interest in this paper.

Next, assume that (13) holds for all $\tau$ and that (13) implies (14) for $\tau=q$. Choosing $m=2 q$ in (11), the left-hand side of (13) becomes

$$
\begin{aligned}
& \sum_{k=1}^{n} a_{k} \mathcal{P}_{k, 2 q} \\
& =\underbrace{c_{i, 1}^{(2 q)} \sum_{k=1}^{n} a_{k} k}_{\substack{=B_{2 q}\left(\hat{x}_{r+n}\right) \\
\text { by (14) and (ii) }}}+\underbrace{c_{i, 3}^{(2 q)} \sum_{k=1}^{n} a_{k} k^{3}+\cdots+c_{i, 2 q-1}^{(2 q)} \sum_{k=1}^{n} a_{k} k^{2 q-1}}_{=0 \text { by (14) and the induction hypothesis }}+c_{i, 2 q+1}^{(2 q)} \sum_{k=1}^{n} a_{k} k^{2 q+1} \\
& =B_{2 q}\left(\hat{x}_{r+n}\right)+c_{i, 2 q+1}^{(2 q)} \sum_{k=1}^{n} a_{k} k^{2 q+1} .
\end{aligned}
$$

The right-hand side of (13) is simply $B_{2 q}\left(\hat{x}_{r+n}\right)$ from which it is clear that

$$
c_{i, 2 q+1}^{(2 q)} \sum_{k=1}^{n} a_{k} k^{2 q+1}=0 .
$$

However, property (iii) in Lemma 7 ensures that $c_{i, 2 q+1}^{(2 q)} \neq 0$. It immediately follows that $\sum a_{k} k^{2 q+1}=0$, hence (14) is also satisfied for $\tau=q+1$. By induction it follows that (13) implies (14) for all $\tau$.

We have thus shown that (14) implies (13) and conversely that (13) implies (14). Hence, they are equivalent.

Theorem 9. Let $\operatorname{SBP}(\tau, 2 s)$ be defined on the grid (1). Then $s \geq \tau$.

Proof. The operator, being of order $\tau$ near the boundaries, must satisfy conditions (13). By Lemma 8, conditions (14) are therefore satisfied and consequently the interior stencil is accurate at least to order $2 \tau$.

The implication of Theorem 9 is clear: There exists no operator $\operatorname{SBP}(\tau, 2 s)$ with $\tau>s$, irrespective of the grid point distribution near the boundary. This is in line with the convergence results observed in [19]. This result is thus a generalization of the classical Theorem 4 to the arbitrary nonuniform grid (1). Note also that Theorem 9 is independent of the dimension of the boundary block and the width of the interior stencil. Thus, wide stencils with additional free parameters, as has been studied in $[17,16]$, cannot be used to allow an increase in boundary accuracy.

5. Generalization of Theorem 5 . In this section we will consider the operator $\operatorname{SBP}(\tau, 2 \tau)$ and generalize Theorem 5 to the arbitrary nonuniform grid (1). To do so, we consider the final condition in (10), i.e., the case when $m=2 \tau$ :

$$
2 \tau \mathbf{1}^{T} \hat{P} \hat{\mathbf{x}}_{1}^{2 \tau-1}=\sum_{k=1}^{n} a_{k} \mathcal{P}_{k, 2 \tau}
$$


Since by Lemma 8 the interior stencil satisfies (14), we may simplify the sum on the right-hand side as follows:

$$
\begin{aligned}
& \sum_{k=1}^{n} a_{k} \mathcal{P}_{k, 2 \tau} \\
& =\underbrace{c_{i, 1}^{(2 q)} \sum_{k=1}^{n} a_{k} k}_{\begin{array}{c}
=B_{2 q}\left(\hat{x}_{r+n}\right) \\
\text { by }(14) \text { and (ii) }
\end{array}}+\underbrace{c_{i, 3}^{(2 \tau)} \sum_{k=1}^{n} a_{k} k^{3}+\cdots+c_{i, 2 \tau-1}^{(2 \tau)} \sum_{k=1}^{n} a_{k} k^{2 \tau-1}}_{=0 \text { by }(14)}+c_{i, 2 \tau+1}^{(2 \tau)} \sum_{k=1}^{n} a_{k} k^{2 \tau+1} \\
& =B_{2 \tau}\left(\hat{x}_{r+n}\right)+c_{i, 2 \tau+1}^{(2 \tau)} \sum_{k=1}^{n} a_{k} k^{2 \tau+1} .
\end{aligned}
$$

Here, we have used properties (i) and (ii) of the polynomial coefficients from Lemma 7. Inserting this into (15) gives

$$
2 \tau \mathbf{1}^{T} \hat{P} \hat{\mathbf{x}}_{1}^{2 \tau-1}=B_{2 \tau}\left(\hat{x}_{r+n}\right)+c_{i, 2 \tau+1}^{(2 \tau)} \sum_{k=1}^{n} a_{k} k^{2 \tau+1} .
$$

Note the similarity between (16) and the accuracy conditions (7) from Lemma 6 that were obtained from the quadrature interpretation of $P$. In fact, if the summation term on the right-hand side vanishes, (16) becomes exactly (7) with $m=2 \tau$. This is the key observation required to generalize Theorem 5 .

Theorem 10. Let $\operatorname{SBP}(\tau, 2 \tau)$ be based on the diagonal norm $P$, and defined on the grid (1). Then $P$ defines a quadrature rule of precisely order $2 \tau$.

Proof. Lemma 6 ensures that $P$ defines a quadrature rule of order at least $2 \tau$. Now, assume that $P$ is accurate to order $2 \tau+1$, i.e., (7) is satisfied also for $m=2 \tau$;

$$
2 \tau \mathbf{1}^{T} \hat{P} \hat{\mathbf{x}}_{1}^{2 \tau-1}=B_{2 \tau}\left(\hat{x}_{r+n}\right) .
$$

However, $P$ also satisfies condition (16). Clearly (16) and (17) are simultaneously satisfied if and only if

$$
c_{i, 2 \tau+1}^{(2 \tau)} \sum_{k=1}^{n} a_{k} k^{2 \tau+1}=0 .
$$

Moreover, property (iii) in Lemma 7 ensures that $c_{i, 2 \tau+1}^{(2 \tau)} \neq 0$. Thus, we must have

$$
\sum_{k=1}^{n} a_{k} k^{2 \tau+1}=0 .
$$

However, by (5) this is precisely the condition required for the interior stencil to be at least of order $2 \tau+2$, which violates Definition 3 of $\operatorname{SBP}(\tau, 2 \tau)$. Therefore, (17) cannot be satisfied, which implies that the quadrature is of precisely order $2 \tau$.

Theorem 10 generalizes Theorem 5 to the arbitrary nonuniform grid (1). In the literature, the operator $\operatorname{SBP}(\tau, 2 s)$ is almost exclusively chosen such that $s=\tau$; hence Theorem 10 is usually enforced in practice.

6. Conclusion. Finite difference operators on SBP form often utilize a diagonal norm based structure in order to guarantee stability for variable coefficient problems. A well-known disadvantage of the diagonal norm SBP operators when used on uniform 
grids is that near boundaries, the formal order of accuracy is at most half that of the interior stencil. Even though many attempts have been made at finding ways to increase the order at the boundaries, they have so far been unsuccessful. A recent and particularly promising idea is to utilize nonuniform and nonconforming grid point distributions near the domain boundaries in order to achieve a smaller truncation error.

In this paper we have shown that the classical result for uniform grids extends to the nonuniform and nonconforming situation. In other words, a diagonal norm based SBP operator of order $\tau$ must have an interior stencil of order at least $2 \tau$, irrespective of the grid point distribution near the boundary. Further, we have shown that if the interior stencil is precisely of order $2 \tau$, then so is the order of accuracy of $P$ when seen as a quadrature rule. The problem of improving the order of accuracy of diagonal norm based SBP operators can consequently not be addressed by turning to nonuniform and nonconforming grids. Further, it follows from the results in this paper that the formal order of accuracy cannot be increased by either expanding the size of the boundary blocks or utilizing wider interior stencils.

Appendix A. Bernoulli polynomials. The Bernoulli polynomials are defined through the generating function

$$
F(t, x)=\frac{t \exp (x t)}{\exp (t)-1}=\sum_{n=0}^{\infty} B_{n}(x) \frac{t^{n}}{n !} .
$$

Explicitly, the $n$th Bernoulli polynomial may be written

$$
B_{n}(x)=\sum_{k=0}^{n}\left(\begin{array}{l}
n \\
k
\end{array}\right) b_{n-k} x^{k},
$$

where $b_{n-k}$ are the Bernoulli numbers. We will make use of the well-known translation property

$$
B_{n}(x+y)=\sum_{k=0}^{n}\left(\begin{array}{l}
n \\
k
\end{array}\right) B_{k}(x) y^{n-k}
$$

which is easily obtained from (18). Further, for a positive integer $k$, the Bernoulli polynomials satisfy the difference formula

$$
\frac{B_{n}(x+k)-B_{n}(x)}{n}=\sum_{i=0}^{k-1}(x+i)^{n-1} .
$$

Appendix B. Proof of Lemma 7. The purpose of this appendix is to provide the details required to prove Lemma 7 . This encompasses two things: proving the polynomial equality (10), and showing the properties (i), (ii), and (iii) of the polynomial coefficients.

B.1. The polynomial equality (10). We begin by reconsidering the first accuracy condition in Definition 2 satisfied by the SBP operator. Restricting our attention to the $r+n$ rows near the left boundary, it follows from the structure of $P$ and $Q$ that this condition can be rewritten as

$$
\hat{Q} \hat{\mathbf{x}}_{1}^{j}+S \hat{\mathbf{x}}_{2}^{j}=j \hat{P} \hat{\mathbf{x}}_{1}^{j-1}, \quad j=0, \ldots, \tau,
$$


where we have introduced the $(r+n) \times(r+n)$ matrices $\hat{Q}$ and $\hat{P}$ to denote the boundary blocks of $Q$ and $\Delta x^{-1} P$, respectively (see (3) and (6)). Here, $S$ takes the form

$$
S=\left(\begin{array}{cc}
0 & 0 \\
\hat{S} & 0
\end{array}\right)
$$

where

$$
\hat{S}=\left(\begin{array}{cccc}
a_{n} & & & 0 \\
a_{n-1} & a_{n} & & \\
\vdots & & \ddots & \\
a_{1} & \ldots & & a_{n}
\end{array}\right)
$$

Further, we have partitioned the grid as $\mathbf{x}^{T}=\Delta x\left(\hat{\mathbf{x}}_{1}^{T}, \hat{\mathbf{x}}_{2}^{T}\right)$, where

$$
\begin{aligned}
& \hat{\mathbf{x}}_{1}=\left(\hat{x}_{0}, \hat{x}_{1}, \ldots, \hat{x}_{r+n-1}\right)^{T}, \\
& \hat{\mathbf{x}}_{2}=\left(\hat{x}_{r+n-1}+1, \hat{x}_{r+n-1}+2, \ldots\right)^{T} .
\end{aligned}
$$

From (21), we derive a new set of compatibility conditions analogous to (2). First, set $i=j=0$. Multiplying (21) from the left by $\left(\hat{\mathbf{x}}_{1}^{0}\right)^{T}$, then adding the transpose of the result yields

$$
0=-1+\left(\hat{\mathbf{x}}_{1}^{0}\right)^{T} S \hat{\mathbf{x}}_{2}^{0}+\left(\hat{\mathbf{x}}_{2}^{0}\right)^{T} S^{T} \hat{\mathbf{x}}_{1}^{0}=-1+2 \mathbf{1}_{n}^{T} \hat{S} \mathbf{1}_{n}=-1+2 \sum_{k=1}^{n} a_{k} k,
$$

where $\mathbf{1}_{n}$ is the all-ones vector of dimension $n$. However, by (5), $\sum a_{k} k=1 / 2$ for all consistent interior stencils. Hence, the equality (21) is satisfied in the special case $i=j=0$. Thus, we will not deliberate that case further.

For all other viable choices of $i$ and $j$, multiplying (21) from the left by $\left(\hat{\mathbf{x}}_{1}^{i}\right)^{T}$, then adding the result with $i$ and $j$ swapped yields

$$
(i+j) \mathbf{1}^{T} \hat{P} \hat{\mathbf{x}}_{1}^{i+j-1}=\left(\hat{\mathbf{x}}_{1}^{i}\right)^{T} S \hat{\mathbf{x}}_{2}^{j}+\left(\hat{\mathbf{x}}_{1}^{j}\right)^{T} S \hat{\mathbf{x}}_{2}^{i}
$$

with $0 \leq i, j \leq \tau$ and $1 \leq i+j \leq 2 \tau$. Here, $\mathbf{1}=\hat{\mathbf{x}}_{1}^{0}$ is the all-ones vector of dimension $r+n$. To obtain (22) we have used the fact that

$$
\left(\hat{\mathbf{x}}_{1}^{i}\right)^{T}\left(\hat{Q}+\hat{Q}^{T}\right) \hat{\mathbf{x}}_{1}^{j}=-\left(\hat{\mathbf{x}}_{1}^{i}\right)^{T}\left(e_{a}^{T} e_{a}\right) \hat{\mathbf{x}}_{1}^{j}=-\left(e_{a} \hat{\mathbf{x}}_{1}^{i}\right)^{T}\left(e_{a} \hat{\mathbf{x}}_{1}^{j}\right)=-a^{i+j}=0
$$

since $a=0$ and $i+j \neq 0$.

In component form, the right-hand side of (22) becomes

$$
\begin{aligned}
\left(\hat{\mathbf{x}}_{1}^{i}\right)^{T} S \hat{\mathbf{x}}_{2}^{j}+\left(\hat{\mathbf{x}}_{1}^{j}\right)^{T} S \hat{\mathbf{x}}_{2}^{i} & =\sum_{k=1}^{n} a_{k} \sum_{l=0}^{k-1}\left\{\left(\hat{x}_{r+n}+l-k\right)^{i}\left(\hat{x}_{r+n}+l\right)^{j}\right. \\
& \left.+\left(\hat{x}_{r+n}+l-k\right)^{j}\left(\hat{x}_{r+n}+l\right)^{i}\right\} \\
& =\sum_{k=1}^{n} a_{k}\left(J_{i, m}+J_{m-i, m}\right),
\end{aligned}
$$

where, for simplicity, we have introduced $m=i+j$. From the limits of $i, j$, and $m$ we thus have $\max \{0, m-\tau\} \leq i \leq \min \{m, \tau\}$. Our goal is to show that (23) is equal to the right-hand side of (10). In (23), we have defined

$$
J_{i, m}(k)=\sum_{l=0}^{k-1}\left(\hat{x}_{r+n}+l-k\right)^{i}\left(\hat{x}_{r+n}+l\right)^{m-i} .
$$

Copyright $@$ by SIAM. Unauthorized reproduction of this article is prohibited. 
A binomial expansion of the first factor in $J_{i, m}$ gives

$$
\begin{aligned}
J_{i, m} & =\sum_{l=0}^{k-1}\left[\sum_{h=0}^{i}\left(\begin{array}{c}
i \\
h
\end{array}\right)(-k)^{h}\left(\hat{x}_{r+n}+l\right)^{i-h}\right]\left(\hat{x}_{r+n}+l\right)^{m-i} \\
& =\sum_{h=0}^{i}\left(\begin{array}{c}
i \\
h
\end{array}\right)(-k)^{h} \sum_{l=0}^{k-1}\left(\hat{x}_{r+n}+l\right)^{m-h} \\
& =\sum_{h=0}^{i}\left(\begin{array}{l}
i \\
h
\end{array}\right) \frac{(-k)^{h}}{m-h+1}\left[B_{m-h+1}\left(\hat{x}_{r+n}+k\right)-B_{m-h+1}\left(\hat{x}_{r+n}\right)\right],
\end{aligned}
$$

where we have used the difference formula (20) for the Bernoulli polynomials in the final equality. Expanding the first Bernoulli polynomial using (19) and collecting terms results in

$$
J_{i, m}=\sum_{h=0}^{i}\left(\begin{array}{l}
i \\
h
\end{array}\right) \frac{(-1)^{h}}{m-h+1} \sum_{\nu=0}^{m-h}\left(\begin{array}{c}
m-h+1 \\
\nu
\end{array}\right) B_{\nu}\left(\hat{x}_{r+n}\right) k^{m-\nu+1} .
$$

Now consider $J_{m-i, m}$ in (23). From (24) we have

$J_{m-i, m}=\sum_{l=0}^{k-1}\left(\hat{x}_{r+n}+l-k\right)^{m-i}\left(\hat{x}_{r+n}+l\right)^{i}=\sum_{l=0}^{k-1}\left(\hat{x}_{r+n}+l-k\right)^{m-i}\left(\hat{x}_{r+n}+l-k+k\right)^{i}$,

where we have added and subtracted $k$ in the second factor. Binomially expanding the second factor gives

$$
\begin{aligned}
J_{m-i, m} & =\sum_{l=0}^{k-1}\left(\hat{x}_{r+n}+l-k\right)^{m-i} \sum_{h=0}^{i}\left(\begin{array}{l}
i \\
h
\end{array}\right) k^{h}\left(\hat{x}_{r+n}+l-k\right)^{i-h} \\
& =\sum_{h=0}^{i}\left(\begin{array}{l}
i \\
h
\end{array}\right) k^{h} \sum_{l=0}^{k-1}\left(\hat{x}_{r+n}+l-k\right)^{m-h} \\
& =\sum_{h=0}^{i}\left(\begin{array}{l}
i \\
h
\end{array}\right) \frac{k^{h}}{m-h+1}\left[B_{m-h+1}\left(\hat{x}_{r+n}\right)-B_{m-h+1}\left(\hat{x}_{r+n}-k\right)\right],
\end{aligned}
$$

where as before, we have used the difference formula (20) in the final equality. Expanding the second Bernoulli polynomial using (19), inserting the result into (26), and collecting terms results in

$$
J_{m-i, m}=-\sum_{h=0}^{i}\left(\begin{array}{l}
i \\
h
\end{array}\right) \frac{(-1)^{h}}{m-h+1} \sum_{\nu=0}^{m-h}\left(\begin{array}{c}
m-h+1 \\
\nu
\end{array}\right) B_{\nu}\left(\hat{x}_{r+n}\right) k^{m-\nu+1}(-1)^{m-\nu+1} .
$$

Combining $J_{i, m}$ and $J_{m-i, m}$, we thus have

$$
\begin{aligned}
J_{i, m} & +J_{m-i, m} \\
= & \sum_{h=0}^{i}\left(\begin{array}{l}
i \\
h
\end{array}\right) \frac{(-1)^{h}}{m-h+1} \sum_{\nu=0}^{m-h}\left(\begin{array}{c}
m-h+1 \\
\nu
\end{array}\right) B_{\nu}\left(\hat{x}_{r+n}\right)\left[1-(-1)^{m-\nu+1}\right] k^{m-\nu+1} .
\end{aligned}
$$

Copyright $@$ ㅇ by SIAM. Unauthorized reproduction of this article is prohibited. 
Note that the binomial coefficient $\left(\begin{array}{l}i \\ h\end{array}\right)=0$ for $h>i$. We may therefore extend the limit of the first sum in (27) to $h=m$ without changing its value. If we then exchange the two sums, we obtain

$$
\begin{aligned}
J_{i, m} & +J_{m-i, m} \\
= & \sum_{\nu=0}^{m} B_{\nu}\left(\hat{x}_{r+n}\right)\left[1-(-1)^{m-\nu+1}\right] \sum_{h=0}^{m-\nu}\left(\begin{array}{l}
i \\
h
\end{array}\right) \frac{(-1)^{h}}{m-h+1}\left(\begin{array}{c}
m-h+1 \\
\nu
\end{array}\right) k^{m-\nu+1} .
\end{aligned}
$$

Finally, we reverse and shift the order of summation in the sum over $\nu$ so that $\nu$ is replaced with $m-\nu+1$ everywhere, and we obtain

$$
J_{i, m}(k)+J_{m-i, m}(k)=\mathcal{P}_{k, m}^{(i)},
$$

where

$$
\mathcal{P}_{k, m}^{(i)}=\sum_{\nu=1}^{m+1}\left[1-(-1)^{\nu}\right] B_{m-\nu+1}\left(\hat{x}_{r+n}\right) \sum_{h=0}^{\nu-1}\left(\begin{array}{l}
i \\
h
\end{array}\right) \frac{(-1)^{h}}{m-h+1}\left(\begin{array}{l}
m-h+1 \\
m-\nu+1
\end{array}\right) k^{\nu} .
$$

Inserting (28) into (23) and recalling that (23) in the right-hand side of (22) give the desired result (10).

B.2. Properties of the polynomial coefficients. It is clear from (29) that the polynomial coefficients $c_{i, \nu}^{(m)}$ appearing in Lemma 7 are indeed given by

$$
c_{i, \nu}^{(m)}=\left[1-(-1)^{\nu}\right] B_{m-\nu+1}\left(\hat{x}_{r+n}\right) \sum_{h=0}^{\nu-1}\left(\begin{array}{l}
i \\
h
\end{array}\right) \frac{(-1)^{h}}{m-h+1}\left(\begin{array}{l}
m-h+1 \\
m-\nu+1
\end{array}\right) .
$$

Recall that we must prove the following three properties:

(i) Whenever $\nu$ is even, $c_{i, \nu}^{(m)}=0$.

(ii) The first coefficient is given by $c_{i, 1}^{(m)}=2 B_{m}\left(\hat{x}_{r+n}\right)$.

(iii) Whenever $m$ is even, $c_{i, m+1}^{(m)} \neq 0$.

Property (i) follows trivially from (12). To prove property (ii) we set $\nu=1$ in (12) and obtain

$$
c_{i, 1}^{(m)}=2 B_{m}\left(\hat{x}_{r+n}\right)\left(\begin{array}{l}
i \\
0
\end{array}\right) \frac{1}{m+1}\left(\begin{array}{c}
m+1 \\
m
\end{array}\right)=2 B_{m}\left(\hat{x}_{r+n}\right) .
$$

Finally, to prove (iii) consider any even $m$ and let $\nu=m+1$. Then, (12) gives

$$
\begin{aligned}
c_{i, m+1}^{(m)} & =2 B_{0}\left(\hat{x}_{r+n}\right) \sum_{h=0}^{m}\left(\begin{array}{l}
i \\
h
\end{array}\right) \frac{(-1)^{h}}{m-h+1}\left(\begin{array}{c}
m-h+1 \\
0
\end{array}\right) \\
& =2 \sum_{h=0}^{i}\left(\begin{array}{l}
i \\
h
\end{array}\right) \frac{(-1)^{h}}{m-h+1} \\
& =2 \sum_{h=0}^{i}\left(\begin{array}{l}
i \\
h
\end{array}\right)(-1)^{h} \int_{0}^{1} x^{m-h} \mathrm{~d} x \\
& =2 \int_{0}^{1} x^{m-i} \sum_{h=0}^{i}\left(\begin{array}{l}
i \\
h
\end{array}\right)(-1)^{h} x^{i-h} \mathrm{~d} x
\end{aligned}
$$

Copyright $\odot$ by SIAM. Unauthorized reproduction of this article is prohibited. 


$$
\begin{aligned}
& =2 \int_{0}^{1} x^{m-i}(x-1)^{i} \mathrm{~d} x \\
& =2(-1)^{i} \int_{0}^{1} x^{m-i}(1-x)^{i} \mathrm{~d} x .
\end{aligned}
$$

In the second equality, we have used the facts that $B_{0}\left(\hat{x}_{r+n}\right)=1$ and $m \geq i$, and we applied the binomial expansion theorem in the fifth equality. Since the final integral is positive it follows that $c_{i, m+1}^{(m)} \neq 0$. This proves property (iii) of the polynomial coefficients and thus completes the proof of Lemma 7.

\section{REFERENCES}

[1] B. K. Alpert, Hybrid Gauss-trapezoidal quadrature rules, SIAM J. Sci. Comput., 20 (1999), pp. $1551-1584$

[2] M. Carpenter and D. Gottlieb, Spectral methods on arbitrary grids, J. Comput. Phys., 129 (1996), pp. $74-86$.

[3] M. H. Carpenter, J. Nordström, and D. Gottlieb, A stable and conservative interface treatment of arbitrary spatial accuracy, J. Comput. Phys., 148 (1999), pp. 341-365.

[4] D. C. Del Rey Fernández, P. D. Boom, And D. ZingG, A generalized framework for nodal first derivative Summation-by-Parts operators, J. Comput. Phys., 266 (2014), pp. 214-239.

[5] D. C. Del Rey Fernández, P. D. Boom, and D. W. ZingG, Corner-corrected diagonal-norm Summation-by-Parts operators for the first derivative with increased order of accuracy, J. Comput. Phys., 330 (2017), pp. 902-923.

[6] T. Fisher AND M. CARPENTER, High-order entropy stable finite difference schemes for nonlinear conservation laws: Finite domains, J. Comput. Phys., 252 (2013), pp. 518-557.

[7] L. Friedrich, D. C. Fernández, A. R. Winters, G. J. Gassner, D. W. ZingG, and J. Hicken, Conservative and Stable Degree Preserving SBP Finite Difference Operators for Non-conforming Meshes, preprint, arXiv:1611.00979, 2016.

[8] G. J. Gassner, A skew-symmetric discontinuous Galerkin spectral element discretization and its relation to SBP-SAT finite difference methods, SIAM J. Sci. Comput., 35 (2013), pp. A1233-A1253.

[9] G. J. Gassner, A. R. Winters, and D. A. Kopriva, Split form nodal discontinuous Galerkin schemes with Summation-by-Parts property for the compressible Euler equations, J. Comput. Phys., 327 (2016), pp. 39-66.

[10] B. Gustafsson, The convergence rate for difference approximations to mixed initial boundary value problems, Math. Comput., 29 (1975), pp. 396-406.

[11] B. Gustafsson, The convergence rate for difference approximations to general mixed initialboundary value problems, SIAM J. Numer. Analy., 18 (1981), pp. 179-190.

[12] J. E. Hicken, D. C. Del Rey Fernández, And D. W. ZingG, Multidimensional summationby-parts operators: General theory and application to simplex elements, SIAM J. Sci. Comput., 38 (2016), pp. A1935-A1958.

[13] J. E. Hicken And D. W. ZingG, Summation-by-Parts operators and high-order quadrature, J. Comput. Appl. Math., 237 (2013), pp. 111-125.

[14] H.-O. Kreiss AND G. Scherer, Finite element and finite difference methods for hyperbolic partial differential equations, in Mathematical Aspects of Finite Elements in Partial Differential Equations, Academic Press, New York, 1974, pp. 195-212.

[15] H.-O. Kreiss And G. Scherer, On the Existence of Energy Estimates for Difference Approximations of Hyperbolic Systems, Technical report, Department of Scientific Computing, Uppsala University, Sweden, 1977.

[16] V. Linders, M. Kupiainen, and J. Nordström, Summation-by-parts operators with minimal dispersion error for coarse grid flow calculations, J. Comput. Phys., 340 (2017), pp. $160-176$.

[17] V. Linders AND J. NORDSTRÖM, Uniformly best wavenumber approximations by spatial central difference operators, J. Comput. Phys., 300 (2015), pp. 695-709.

[18] T. Lundquist and J. Nordström, On the Suboptimal Accuracy of Summation-by-Parts Schemes with Non-conforming Block Interfaces, Technical Report LiTH-MAT-R-2015/16SE, Linköping University, Sweden, 2015.

[19] K. Mattsson, M. Almquist, and M. Carpenter, Optimal diagonal-norm SBP operators, J. Comput. Phys., 264 (2014), pp. 91-111. 
[20] K. MattSSON AND M. CARPENTER, Stable and accurate interpolation operators for high-order multiblock finite difference methods, SIAM J. Sci. Comput., 32 (2010), pp. 2298-2320.

[21] K. Mattsson and J. Nordström, Summation-by-parts operators for finite difference approximations of second derivatives, J. Comput. Phys., 199 (2004), pp. 503-540.

[22] J. Nordström, Conservative finite difference formulations, variable coefficients, energy estimates and artificial dissipation, J. Sci. Comput., 29 (2006), pp. 375-404.

[23] H. Ranocha, P. ÖfFner, And T. Sonar, Summation-by-parts operators for correction procedure via reconstruction, J. Comput. Phys., 311 (2016), pp. 299-328.

[24] B. Strand, Summation-by-parts for finite difference approximations for $d / d x$, J. Comput. Phys., 110 (1994), pp. 47-67.

[25] M. SvÄRD, On coordinate transformations for summation-by-parts operators, J. Sci. Comput., 20 (2004), pp. 29-42.

[26] M. SvÄRD AND J. NoRDSTRÖM, On the order of accuracy for difference approximations of initial-boundary value problems, J. Comput. Phys., 218 (2006), pp. 333-352.

[27] M. Svärd AND J. NordSTRÖm, Review of summation-by-parts schemes for initial-boundaryvalue problems, J. Comput. Phys., 268 (2014), pp. 17-38.

[28] M. SväRd AND J. NoRdSTRÖM, Response to "Convergence of summation-by-parts finite difference methods for the wave equation," J. Sci. Comput., 74 (2017), pp. 1188-1192, https://doi.org/10.1007/s10915-017-0468-x.

Copyright (C) by SIAM. Unauthorized reproduction of this article is prohibited. 